Higher order connections on Lie groupoid: application to materials

\author{
Petr Vašik
}




\title{
HIGHER ORDER CONNECTIONS ON LIE GROUPOID: APPLICATION TO MATERIALS
}

\author{
PETR VAŠÍK
}

\begin{abstract}
We present a possibility of material representation by a higher order connection on a Lie groupoid instead of a representation by principal connections on a principal bundle. We also prove some interesting properties of higher order connections on a Lie groupoid induced by principal connections with respect to the material setting.
\end{abstract}

2000 Mathematics Subject Classification: 58A20,53C05

Keywords: Lie groupoid, higher order connection

\section{INTRODUCTION}

We present a method of deciding whether the constitutional equations of certain material are equivalent. We deal with a material whose representation is given by different isomorphic principal bundles with conjugate structure groups and by means of a Lie groupoid associated to these settings we compare the connections induced by constitutive equations on these principal bundles. We use the notion of semiholonomity of a higher order connection on a Lie groupoid, which was studied in [5]. We also compare the results with those obtained in [3] for connections on general bundles.

\section{LIE GROUPOIDS}

We start with a definition of a general groupoid, see [2].

Definition 1. A groupoid is defined as a pair $(\Phi, B)$ of a total set $\Phi$ and a base set $B$ endowed with two submersions

$$
\alpha: \Phi \rightarrow B \text { and } \beta: \Phi \rightarrow B
$$

called the source and target maps, respectively, and a binary operation defined for those ordered pairs $(y, z) \in \Phi \times \Phi$ such that $\alpha(z)=\beta(y)$. This operation must satisfy the following properties:

(1) Associativity:

$$
(x y) z=x(y z),
$$

whenever the products are defined; 
(2) Existence of identities: for each $b \in B$ there exists an element $\operatorname{id}_{b} \in \Phi$, called the identity at $b$, such that $z \operatorname{id}_{b}=z$ whenever $\alpha(z)=b$, and $\operatorname{id}_{b} z=z$ whenever $\beta(z)=b$;

(3) Existence of inverse: for each $z \in \Phi$ there exists a (unique) inverse $z^{-1}$ such that

$$
z z^{-1}=\mathrm{id}_{\beta(z)} \text { and } z^{-1} z=\mathrm{id}_{\alpha(z)} .
$$

Definition 2. A groupoid $(\Phi, B)$ is said to be transitive if for each pair of points $a, b \in B$ there exists at least one element $z \in \Phi$ such that

$$
\alpha(z)=a \text { and } \beta(z)=b .
$$

Definition 3. A groupoid $(\Phi, B)$ is a Lie groupoid if the total set $\Phi$ and the base set $B$ are differentiable manifolds, the projections $\alpha, \beta$ are smooth and so are the operations of composition and of inverse.

Remark 1. Note that any transitive Lie groupoid induces naturally a set of isomorphic principal bundles with mutually conjugate structure groups, more precisely for every Lie groupoid $\Phi$ and every $x \in B$

$$
\Phi_{x}:=\{\theta \in \Phi \mid \alpha(\theta)=x\}
$$

is a principal bundle, whose structure group $G_{x}$ is the isotropy group of $\Phi$ over $x$. On the other hand, any principal bundle $\pi: P \rightarrow B$ with structure group $G$ induces a Lie groupoid $P P^{-1}:=(P \times P) / G$, where the equivalence class is given by $p q^{-1}:=(p, q) \sim(p g, q g)$ for $p, g \in P$ and $g \in G$, see e. g. [2,4] for further details. Let us just note that the projections in this case are then defined by

$$
\alpha\left(p q^{-1}\right)=\pi(p) \text { and } \beta\left(p q^{-1}\right)=\pi(q) .
$$

\section{CONNECTIONS ON LIE GROUPOIDS}

First, following the paper [5], let us identify the jet prolongation $J^{r}(B \times \Phi)$ of a fibered manifold $B \times \Phi \rightarrow B$ with the set $J^{r}(B, \Phi)$ of $r$-jets of mappings $B \rightarrow \Phi$. On the nonholonomic jet prolongation $\widetilde{J}^{r}(B \times \Phi)$ for integers $r \geq q \geq 0$ we denote by $\pi_{q}^{r}$ the target surjection $\pi_{q}^{r}: \widetilde{J}^{r}(B, \Phi) \rightarrow \widetilde{J}^{q}(B, \Phi)$ with $\pi_{r}^{r}$ being the identity on $\widetilde{J}^{r}(B, \Phi)$. Together with $\pi_{q}^{r}$ we have also the surjections $J^{k} \pi_{q-k}^{r-k}: \widetilde{J}^{r}(B, \Phi) \rightarrow$ $\tilde{J}^{q}(B, \Phi)$. Then the following holds, [5].

Lemma 1. The element $X \in \widetilde{J}^{r}(B, \Phi)$ is semiholonomic if and only if

$$
\left(J^{k} \pi_{q-k}^{r-k}\right)(X)=\pi_{q}^{r}(X) \text { for any integers } 1 \leq k \leq q \leq r .
$$

The space of semiholonomic $r$-jets will be denoted by $\tilde{J}^{r}(B, \Phi)$.

The notion of a higher order connection on a Lie groupoid was established by C. Ehresmann in [1]. Let $\sim: B \rightarrow \Phi$ denote an inclusion of the manifold units into the groupoid and let us consider the projections $\pi_{k}^{r}$ as above. We use the notation of [5]. 
Definition 4. A nonholonomic, semiholonomic or holonomic connection of order $r \geq 1$ on $\Phi$ is a smooth map

$$
\Gamma: B \rightarrow \tilde{J}^{r}(B, \Phi), B \rightarrow \bar{J}^{r}(B, \Phi) \text { or } B \rightarrow J^{r}(B, \Phi),
$$

respectively, satisfying

$$
\pi_{0}^{r} \Gamma=\sim,\left(j^{r} \alpha\right) \Gamma(x)=j_{x}^{r}(u \rightarrow u),\left(j^{r} \beta\right) \Gamma(x)=j_{x}^{r}(u \rightarrow x)
$$

for all $u, x \in B$.

Remark 2. According to [6], let us consider the set

$$
\begin{aligned}
\tilde{Q}^{r}(\Phi)=\left\{X \in \tilde{J}^{r}(B, \Phi) \mid \pi_{0}^{r} X\right. & =\sim(x), \\
& \left.\left(j^{r} \alpha\right) X=j_{x}^{r}(u \rightarrow u),\left(j^{r} \beta\right) X=j_{x}^{r}(u \rightarrow x),(\alpha(X)=x)\right\},
\end{aligned}
$$

where $\alpha$ is the source map. Then $\alpha: \widetilde{Q}^{r}(\Phi) \rightarrow B$ is a fibered manifold and the $r$ th order connections are the sections $B \rightarrow \widetilde{Q}^{r}(\Phi)$.

It is well known that for $r=1$ this corresponds to the standard notion of a connection on any of the principal bundles determined by $\Phi$. Recall that in the language of jet prolongations, a principal connection on a principal bundle is defined as follows. Let us consider a principal bundle $(P, p, B, G)$, where $p: P \rightarrow B$ is a fibered manifold, $G$ is a Lie group and by $r$ we denote the principal right action $r: P \times G \rightarrow P$ and write $r^{g}=r(-, g): P \rightarrow P$ for $g \in G$. We also denote by $r$ the canonical right action $r: J^{1} P \times G \rightarrow J^{1} P$ given by $r^{g}\left(j_{x}^{1} s\right)=j_{x}^{1}\left(r^{g} \circ s\right)$ for all $g \in G$ and $j_{x}^{1} s \in J^{1} P$. A principal connection $\Gamma$ on a principal fiber bundle $P$ with a principal action $r$ is an $r$-equivariant section $\Gamma: P \rightarrow J^{1} P$ of the first jet prolongation $J^{1} P \rightarrow P$.

The above definition together with the constructions mentioned in Remark 1 proves the following claim.

Proposition 1. A principal connection on a principal bundle $P \rightarrow B$ induces naturally a first order connection on the Lie groupoid $P P^{-1}$ and any first order connection on a Lie groupoid $\Phi$ induces a principal connection on the principal bundle $\Phi_{x}$ for any $x \in B$.

The following concept of a construction of higher order connection can be found in [5]. Let now $\xi=\xi^{(1)}: x \rightarrow j_{x}^{1} \xi_{x}$ be a first order connection on $\Phi$ and define for each integer $r \geq 1$ the map

$$
\xi^{(r)}: B \rightarrow \widetilde{J}^{r}(B, \Phi), x \rightarrow j_{x}^{1}\left(u \rightarrow j_{u}^{r-1}\left[\xi_{x}(u)\right]\right) .
$$

Then for any $r$ th order connection $\Gamma$ and first order connection $\xi$ on $\Phi$ the map

$$
\Gamma * \xi: B \rightarrow \widetilde{J}^{r+1}(B, \Phi), x \rightarrow j_{x}^{1} \Gamma \cdot \xi^{(r+1)}(x)
$$

is well defined connection on $\Phi$ of order $r+1$. Note that $\Gamma^{\prime}=\Gamma * \pi_{1}^{r} \Gamma$ is called the prolongation of $\Gamma$. Furthermore, given $r$ first order connections $\xi_{1}, \xi_{2}, \ldots, \xi_{r}$ on $\Phi$, 
we can define recurrently the $r$ th order connection on $\Phi$ as a composition $\xi_{1} * \cdots * \xi_{r}$. On the other hand, given an $r$ th order connection $\Gamma$ on $\Phi$, we can define $r$ first order connections

$$
\xi_{s}=\xi_{s}(\Gamma): B \rightarrow J^{1}(B, \Phi): x \rightarrow\left(j_{1} \pi_{0}^{(s-1)}\right) \pi_{s}^{r} \Gamma(x)
$$

for $s=1, \ldots, r$.

Using this notation, let us mention the classification property of higher order connections on a Lie groupoid $\Phi$, [5].

Theorem 1. If $\Gamma$ is a semiholonomic connection on $\Phi$, then all $\xi_{s}(\Gamma)$ are equal, i.e $\Gamma=\xi * \cdots * \xi$. Moreover, a connection $\xi * \cdots * \xi$ is holonomic if and only if $\xi$ is curvature free.

To recall some further properties of higher order connections on a Lie groupoid $\Phi$ we have to mention the following notions, [5]. Let us denote by $G=G(\Phi)$ the isotropy group bundle and by $L=L(\Phi)$ the isotropy Lie algebra bundle attached to $\Phi$, i. e.

$$
G_{x}=\{\theta \in \Phi \mid \alpha(\theta)=\beta(\theta)=x\} \text { and } L_{x}=T_{\widetilde{x}}\left(G_{x}\right),
$$

where $\tilde{x} \in \Phi$ is an image of $x \in B$ under the inclusion $\sim$. Then the following holds, [5].

Theorem 2. Every second order connection $\Gamma$ on $\Phi$ is uniquely determined by two first order connections $\xi_{1}(\Gamma), \xi_{2}(\Gamma)$ and a linear map

$$
A(\Gamma): T B \otimes T B \rightarrow L(\Phi) .
$$

Now if $\Gamma$ and $\bar{\Gamma}$ are two $r$ th order connections on $\Phi$, we can consider the composition $\bar{\Gamma} \cdot \Gamma^{-1}: x \rightarrow \bar{\Gamma}(x) \cdot \Gamma^{-1}(x)$. To generalize the linear map $A(\Gamma)$ from the previous theorem we put

$$
A(\Gamma)=\Gamma \cdot\left[\xi_{1}(\Gamma) * \cdots * \xi_{r}(\Gamma)\right]^{-1} .
$$

Then the following holds, [5].

Theorem 3. Let $\Gamma$ be an $r$ th order connection on $\Phi$. Then $\Gamma$ is uniquely determined by $\xi_{1}(\Gamma), \ldots, \xi_{r}(\Gamma)$ and $A(\Gamma)$. Moreover, $\Gamma$ is semiholonomic if and only if all $\xi_{s}(\Gamma)$ are equal, and $A(\Gamma)$ is semiholonomic.

Finally, let us recall that two $r$ th order connections $\Gamma, \bar{\Gamma}$ on $\Phi$ are said to be equivalent in the $q$-th order $(1 \leq q<r)$ if

$$
\pi_{q}^{r, C} \Gamma=\pi_{q}^{r, C} \bar{\Gamma}
$$

for all decreasing sequences $C=\left\{r \geq c_{1}>\cdots>c_{r-q} \geq 1\right\}$, where

$$
\pi_{q}^{r, c_{i}} \Gamma=\left(j^{c_{i}} \pi_{q-c_{i}}^{r-c_{i}}\right) \Gamma
$$

Especially they are equivalent in the $(r-1)$-st order if $\bar{\Gamma} \cdot \Gamma^{-1}$ is a section in

$$
L(\Phi) \otimes\left(\stackrel{r}{\otimes} T^{*} B\right) .
$$




\section{Applications to MATERials}

In material sciences, the basic setting is often given in the form of a principal bundle, for the case of so called Cosserat media see [2], endowed with a principal connection obtained from a constitutive equation. Let us consider the following case.

Proposition 2. Let a material admit different settings in the form of $r$ mutually isomorphic principal bundles $\left(P_{i}, B, G_{i}\right)$ with the same base manifold $B$ and with conjugate structure groups $G_{i}$. Let each of the settings be endowed with a material connection $\Gamma_{i}$. The set of settings $\left\{\left(P_{i}, B, G_{i}\right), \Gamma_{i}, i=1, \ldots, r\right\}$ is equivalent to a setting given by a Lie groupoid $\Phi=P_{k} P_{k}^{-1}$ for some $k \in\{1, \ldots, r\}$ and an $r$ th order connection $\Gamma$ on $\Phi$. Moreover, if $\Gamma$ is semiholonomic, then the connections $\Gamma_{i}$ are generated by equivalent constitutive equations.

Proof. According to Remark 1, each principal bundle $\left(P_{i}, B, G_{i}\right)$ induces Lie groupoid $P_{i} P_{i}^{-1}$. Obviously, as the principal bundles are isomorphic with conjugate structure groups, Lie groupoids $P_{i} P_{i}^{-1}$ and $P_{j} P_{j}^{-1}$ are isomorphic for any $i, j \in\{1, \ldots, r\}$. Furthermore, each of the connections $\Gamma_{i}$ corresponds to a connection on the Lie groupoid $P_{i} P_{i}^{-1}$ and thus, up to a Lie groupoid isomorphism, they correspond to $r$ first order connection $\Gamma_{i}$ on a Lie groupoid $P_{k} P_{k}^{-1}$ for certain $k \in\{1, \ldots, r\}$. Then

$$
\Gamma=\Gamma_{1} * \cdots * \Gamma_{r}
$$

According to Theorem 1, if $\Gamma$ is semiholonomic, then all generating connections $\Gamma_{i}$ are equal and thus they were generated by equivalent constitutive equations.

Let us consider the case when the connection $\Gamma$ is not semiholonomic, i.e. the generating connections are not all equal. Then we can consider $r$ ! connections of order $r$ obtained from $r$ first order connections by changing the order in the expression $\Gamma_{1} * \cdots * \Gamma_{r}$. Let us denote such connections by $\bar{\Gamma}^{i}, i \in I=\{1, \ldots, r !\}$. Then the following holds.

Proposition 3. If $\bar{\Gamma}^{i}$ and $\bar{\Gamma}^{j}$ are equivalent in $(r-1)$-st order for some couple $(i, j) \in I \times I, i \neq j$, then there exist at least 2 couples of equal generating connections, i.e. there are 2 couples of equivalent corresponding constitutive equations.

Proof. If $\bar{\Gamma}^{i}$ and $\bar{\Gamma}^{j}$ are equivalent in $(r-1)$-st order, then

$$
\left(j^{k} \pi_{r-k-1}^{r-k}\right) \bar{\Gamma}^{i}=\left(j^{k} \pi_{r-k-1}^{r-k}\right) \bar{\Gamma}^{j}
$$

for $k=0,1, \ldots, r-1$. Particularly,

$$
\pi_{r-1}^{r} \bar{\Gamma}^{i}=\pi_{r-1}^{r} \bar{\Gamma}^{j}
$$

i. e. for the first order generating connections it holds that $\Gamma_{l}^{i}=\Gamma_{l}^{j}$ for $l=$ $1, \ldots, r-1$ and $\Gamma_{r}^{i} \neq \Gamma_{r}^{j}$. But this means from the construction of $\bar{\Gamma}^{i}$ and $\bar{\Gamma}^{j}$ 
that there exist connections $\Gamma_{m}^{i}, \Gamma_{n}^{j}$ different from $\Gamma_{r}^{i}, \Gamma_{r}^{j}$ such that the relations

$$
\Gamma_{r}^{i}=\Gamma_{m}^{i} \text { and } \Gamma_{r}^{j}=\Gamma_{n}^{j}
$$

hold.

By analogous yet more combinatoric considerations we obtain

Proposition 4. If $k$ connections $\bar{\Gamma}^{i_{1}}, \ldots, \bar{\Gamma}^{i_{k}},\left\{i_{1}, \ldots, i_{k}\right\} \subset I$, are equivalent in $(r-1)$ th order, then there exist at least $k$ couples of equal generating connections, i. e. there are $k$ couples of equivalent corresponding constitutive equations.

Let us denote by $\left\langle\Gamma_{1} * \cdots * \Gamma_{k}\right\rangle$ the set of all permutations of $k$ first order connections $\Gamma_{1}, \ldots, \Gamma_{k}$. Then by excluding one of the equal connections and by iterating the process described in Proposition 4 we obtain the following assertion, which reduces the number of material connections to just those corresponding to non-equivalent constitutive equations.

Corollary 1. Let us suppose that there is no pair of connections equivalent in $\left(l_{m}-1\right)-$ st order among the connections $\left\langle\Gamma_{l_{1}} * \cdots * \Gamma_{l_{m}}\right\rangle, l_{1}<l_{2}<\cdots<l_{m}, l_{i} \in$ $\{1, \ldots, r\}, i=1, \ldots, m, m<r$, respectively, and for any $n=m+1, \ldots, r$ there exists a pair of connections equivalent in $(n-1)$ th order. Then the appropriate material representation is given by a Lie groupoid and a nonholonomic connection of order $l_{m}$.

Finally, we show an analogue and a generalization of a result proved in [3]. Indeed, in [3] we handled second order connections on fibered manifolds, while Theorem 3 gives us the possibility to prove similar result for $r$ th order connections on a Lie groupoid $\Phi$. Note that for $r>2$ there is no similar identification of connections on fibered manifolds. First, one can define the relation on the space of $r$ th order nonholonomic connections on $\Phi$, for our purpose we identify such space with $(r+1)$-tuples $\left(\Gamma_{1}, \ldots, \Gamma_{r}, A(\Gamma)\right)$ as in Theorem 3. We say that the elements $\left(\Gamma_{1}, \ldots, \Gamma_{r}, A(\Gamma)\right),\left(\bar{\Gamma}_{1}, \ldots, \bar{\Gamma}_{r}, A(\bar{\Gamma})\right)$ are equivalent if and only if $\Gamma_{1}=$ $\bar{\Gamma}_{1}, \ldots, \Gamma_{r}=\bar{\Gamma}_{r}$. It is easy to see that this is an equivalence relation and we denote by $[\theta]=\left[\left(\Gamma_{1}, \ldots, \Gamma_{r}, A(\Gamma)\right)\right]$ a class of this equivalence. Finally the class $[\theta]$ consists of semiholonomic connections if and only if $\Gamma_{1}=\cdots=\Gamma_{r}$ for any $\left(\Gamma_{1}, \ldots, \Gamma_{r}, A(\Gamma)\right) \in[\theta]$. Furthermore, $A(\Gamma)$ is semiholonomic.

Proposition 5. Let $\Phi$ be a Lie groupoid and $[\theta]$ a class of $r$ th order connections. Then the constitutive equations on a principal bundle $\Phi_{x}$ corresponding to first order connections $\Gamma_{1}, \ldots, \Gamma_{r}$ on $\Phi$ are in the same projective class if and only if $[\theta]$ is semiholonomic.

Proof. If the element $\left[\left(\Gamma_{1}, \ldots \Gamma_{r}, A(\Gamma)\right)\right]$ belongs to $[\theta]$, then from Theorem 3 the semiholonomity is equivalent to the property $\Gamma_{1}=\cdots=\Gamma_{r}$. In particular, $r$ constitutive equations determine $r$ projectively equivalent connections of the first order. 


\section{ACKNOWLEDGEMENT}

The author was supported by the Grant no. FSI-S-11-3.

\section{REFERENCES}

[1] C. Ehresmann, "Sur les connexions d'ordre supérieur," Atti del V Congr. dell'Unione Mat. Ital., pp. 1-3, 1956, Pavia-Torino.

[2] M. Epstein and M. Elzanowski, Material Inhomogeneities and their Evolution: A Geometric Approach, Interaction of Mechanics and Mathematics. Springer, 2007.

[3] J. Hrdina and P. Vašík, "Semiholonomic second order connections associated to material bodies," Journal of Applied Mathematics, vol. 2013, 2013.

[4] I. Koláŕ, P. W. Michor, and J. Slovák, Natural Operations in Differential Geometry. SpringerVerlag, 1993.

[5] G. Virsik, "On the holonomity of higher order connections," Cahiers Topol. Géom. Diff., vol. 12, pp. 197-212, 1971.

[6] G. Virsik, "Total connections in Lie groupoid," Arch. Math., vol. 31, pp. 183-200, 1995.

\section{Author's address}

Petr Vašík

Brno University of Technology, Faculty of Mechanical Engineering, Institute of Mathematics, Technická 2, 61669 Brno, Czech Republic

E-mail address: vasikefme.vutbr.cz 